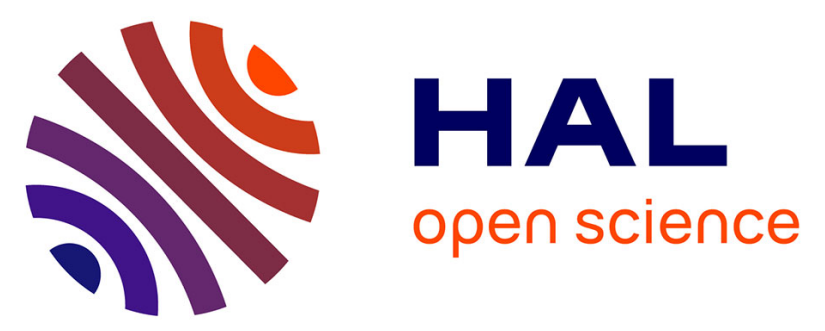

\title{
Analysis of electron transfer between electron irradiated metallic ball and insulators in vacuum: A specific alternative to the mirror method
}

Christelle Guerret-Piécourt, Denyse Juvé, Noël Burais, Daniel Tréheux

\section{- To cite this version:}

Christelle Guerret-Piécourt, Denyse Juvé, Noël Burais, Daniel Tréheux. Analysis of electron transfer between electron irradiated metallic ball and insulators in vacuum: A specific alternative to the mirror method. Journal of Applied Physics, 2002, 92 (12), pp.7425-7433. 10.1063/1.1522477 . hal-00140340

\section{HAL Id: hal-00140340 \\ https://hal.science/hal-00140340}

Submitted on 18 Apr 2007

HAL is a multi-disciplinary open access archive for the deposit and dissemination of scientific research documents, whether they are published or not. The documents may come from teaching and research institutions in France or abroad, or from public or private research centers.
L'archive ouverte pluridisciplinaire HAL, est destinée au dépôt et à la diffusion de documents scientifiques de niveau recherche, publiés ou non, émanant des établissements d'enseignement et de recherche français ou étrangers, des laboratoires publics ou privés. 


\title{
Analysis of electron transfer between electron irradiated metallic ball and insulators in vacuum: A specific alternative to the mirror method
}

\author{
C. Guerret-Piécourt, ${ }^{a)}$ D. Juvé, and D. Tréheux \\ Laboratoire d'Ingénierie et Fonctionnalisation des Surfaces, UMR 5621, Ecole Centrale de Lyon, \\ 36 avenue Guy de Collongue, 69134 Ecully cedex, France \\ N. Burais \\ CEGELY, UMR 5005, Ecole Centrale de Lyon, 36 avenue Guy de Collongue, 69134 Ecully cedex, France
}

(Received 14 June 2002; accepted 26 September 2002)

In order to improve the knowledge of dielectric properties of insulators, we have imagined an original method of characterization of the charge buildup. Electrons of an electron beam are implanted through a metallic ball directly in contact with the insulator in a scanning electron microscope. By calculating and modeling the capacitance and the electrostatic force between the ball and the insulator plane, it has been possible to determine the relationship between the injected charges in the metallic ball and its surface potential. The major role of the dielectric thickness has been evidenced when the insulator is placed on a grounded metallic plane. At high potential values, a dielectric breakdown of the medium surrounding the sphere occurs and electrical charges are transferred from the ball to the dielectric sample. This transfer has been evidenced and quantified in the case of sapphire and quartz. Analytical calculations and numerical simulations using the finite-element method have been performed for interpreting these experimental results. (C) 2002 American Institute of Physics. [DOI: 10.1063/1.1522477]

\section{INTRODUCTION}

Electrical insulators are materials of very high industrial importance. On the one hand, a typical hazardous situation in the electronics, spacecraft, or polymer industry is that of a charged object getting in touch with a semiconductor or an insulator. ${ }^{1-3}$ This situation leads sometimes to breakdown and (or) charge transfer occurring between the two bodies, inducing electrostatic damages ${ }^{4}$ or contact electrification and triboelectrification. ${ }^{5,6}$ The conditions and mechanisms of this charge transfer are not very well known.

On the other hand, it is now well known that macroscopic (electrical, mechanical, etc.) properties of insulators are directly related to the buildup of space charge and the charging ability of the dielectric. ${ }^{7,8}$ Many characterization techniques are currently used to study the charge trapping on defects. ${ }^{9}$ One of them is known as the "scanning electron microscope mirror" (SEMM) method. ${ }^{10-12}$ During the first step of the SEMM experiments, high energetic electrons (30 $\mathrm{keV}$ ) are injected in the insulator, leading to a possible disturbance of the studied sample. To minimize this disturbance and its influence on the characterization of the trapping ability of the material, we have imagined reducing the kinetic energy of the incoming electrons.

Because of both previous interests, we have carefully examined the following problem: What happens if electrons issued from an electron beam of a scanning electron microscope (SEM) are injected through a metallic ball placed in contact with an insulator? Indeed, this experimental configu-

\footnotetext{
a) Author to whom correspondence should be addressed at: LPMI/CURS, BP1155, 64013 PAU Cedex, France; electronic mail: christelle.guerret@ec-lyon.fr
}

ration allows injection by contact of electrons with smaller kinetic energy as in direct SEMM. Moreover, it can simulate what happens in real cases where charges can be injected by contact between metal (electrode) and an insulating material or by triboelectrification. Finally, the system "dielectric plane-metallic sphere," which is currently considered in the field of electrostatic risks, is of great importance. ${ }^{13}$

This article is focused on the interpretation of the results obtained during the electron injection in this experimental arrangement. The experimental system is described in Sec. II. Section III specifies the analytical calculations in the approximation of a semi-infinite plane of dielectric, and the finite-element method simulation in a more realistic configuration. Section IV develops the confrontation of calculation and experimental results. The strong influence of the grounded metallic plane situated under the insulator has been evidenced, thanks to the variation of the capacitance measured with the insulator thickness. Otherwise, the strikingly contribution of the electrostatic force to the mechanical equilibrium of the sphere on the plane, when the system is tilted, has been reported. At a high surface potential of the metallic ball, a slope break of the curve relating the surface potential with the injected charge can be explained either by a dielectrical breakdown due to the electric field exceeding the dielectric strength of the medium ${ }^{3}$ (in our case, secondary vacuum), or by a transfer of electrical charges between the metallic ball and the insulator. An effective transfer of electrons between the metal and the dielectrical plane has been evidenced when the potential of the ball is strong enough, however, the breakdown electric discharge of the medium can be the way of this charge transfer. These points will be discussed in Sec. V. 


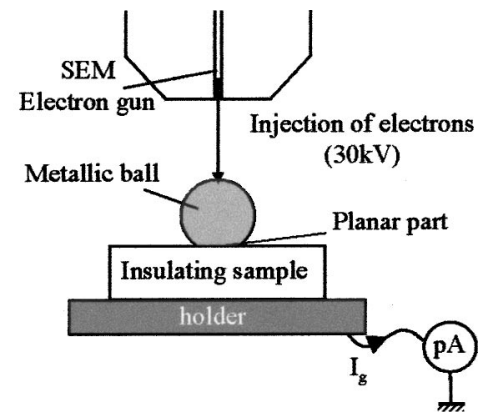

(a)

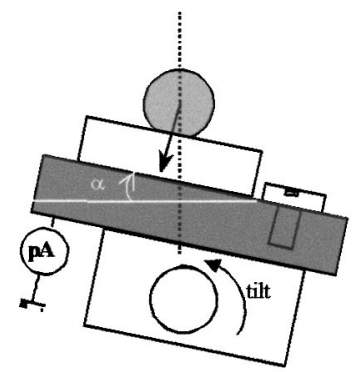

(b)

FIG. 1. Experimental system: (a) Experimental modification of the SEMM method for the injection through a metallic ball. (b) Tilt of the sample for mechanical evaluation of the electrostatic force.

\section{EXPERIMENT}

\section{A. Experimental configuration}

As shown in Fig. 1(a), approximate (metallic) sphereplane (insulator) geometry has been chosen. More precisely, the metallic ball, with a radius of about $1.74 \mathrm{~mm}$, had a plane part for easier manipulation, and the dielectric sample was a parallelepipedal one. The width and length of the dielectric parallelepiped were about $20 \mathrm{~mm}$ and the thickness was varied between $1 \mathrm{~mm}$ and $5 \mathrm{~mm}$ for the experiments. The sphere-parallelepiped system was put on a metallic plane, connected to the ground like the SEM chamber. It is possible to tilt the system in the aim of mechanical equilibrium measure [Fig. 1(b)].

\section{B. Materials}

The tested insulators included very pure monocrystalline aluminas (sapphires) and, for comparison with the analytical and numerical calculations in the function of the dielectric constant values, quartz monocrystal, oriented along the piezoelectric axis. The sapphires were annealed at $1700^{\circ} \mathrm{C}$ in air during $24 \mathrm{~h}$ to control their ability to trap charges. ${ }^{14}$ The sphere was a ball of AI52100 steel. Before the tests, the metallic ball was cleaned in solvent, then the insulating sample and the ball were annealed together, in a vacuum, in the SEM chamber to minimize the superficial contamination.

The secondary-electron emission ratio for the metallic ball (measured in preliminary experiment) was about $36 \%$ for an incident energy of $30 \mathrm{keV}$ of the primary-electron beam. During all of the experiments, the maximum value reached by the surface potential of the ball was about $9 \mathrm{keV}$. It was corresponding to a minimum initial kinetic energy of the incident electrons of about $21 \mathrm{keV}$. So, for the range (30-20 keV) of primary energy obtained during the injection in the metallic sphere the secondary-electron emission can be considered as constant. ${ }^{15}$

\section{Measure of the surface potential of the metallic sphere}

In the original SEMM Method, the insulating sample is directly irradiated with the electron beam of the SEM at a high voltage $(30 \mathrm{kV})$. During the high-voltage irradiation, a well known quantity of charges is implanted and trapped in the dielectric, leading to an electrical field in the vacuum chamber of the SEM. If the sample is observed later, with a lower-energy electron beam (100 to $3000 \mathrm{eV}$ ), the electrical field can be strong enough to deflect the electrons in the same manner as a convex mirror does with light. As a consequence, a "mirror" image is given on the screen that displays a distorted view of the SEM chamber. The amount of trapped charge can be evaluated from the geometrical characteristics of the mirror image by using an electrostatic law. ${ }^{10-12}$ In this particular case (no leakage current, strong trapping, etc.), the trapped charge can also be evaluated by measuring the ground current, due to the influence charge effect. $^{16}$

In the sphere-plane system, a similar procedure is used to evaluate the surface potential $V_{s}$ of the metallic sphere after injection. Due to the insulator presence under the metallic ball, charges injected in the sphere are blocked. The observation at low voltage of the ball leads also to the observation of a "mirror effect." At the beginning of the observation of the mirror, the accelerating potential $V_{a}$ of the electrons, is smaller than the surface potential of the metallic sphere. All the electrons of the lecture beam are deviated on the equipotential, following the Rutherford scattering law, ${ }^{10}$ so that trajectories of the electrons are deflected depending on their incident angle, and they can be backscattered to the upper pieces of the SEM chamber, leading to the mirror image. This experiment is repeated with increasing values of the accelerating potential $V_{a}$, until the beam has reached the ball surface for $V_{a}=V_{s}$. Experimentally, for this value of $V_{a}$, the mirror image abruptly lost its standard shape. In the following, the value of the surface potential of the sphere after injection is experimentally determined thanks to the appearance of this loss of shape, it will be noted $V_{l s}$.

\section{Important remark}

It is important to note that each point of the experimental curves corresponds to a complete experiment. Charges have always been injected in one go. The results have been completely different in the case of multiple successive injections, but they will not be presented in this article.

\section{MODELIZATION}

For a better understanding of the experimental results, the analytical calculation of the simplified system of a metallic sphere lying on an infinite dielectric plane will be considered. However, it has been found that the accuracy of the approximation is only sufficient for a very thick dielectrical 
sample. For a thinner insulator, the presence of the grounded metallic plane has a major influence, which has been evidenced with the finite-element model because of too complex analytical ones.

\section{A. Analytical model}

For the analytical calculation, we have supposed that the dielectric slab is thick and wide enough to be regarded as a semi-infinite plane of the insulator. The analytical calculation did not take into account the contribution of the metallic plane placed under the dielectric, so it was used as a validation of the numerical calculation in the case of a very thick insulating sample. It has been also useful for understanding the experimental results for high quantities of injected charge, when charges are supposed to be transferred from the metallic ball to the dielectric.

The sphere-plane system has been studied by Durand in the case of a metallic ball and metallic plane at a distance $h$, a long time ago. ${ }^{17}$ However, in the case of contact between a sphere and a dielectric plane, it has not been possible to directly use his expression. Similar to the recent work of Bacchetta in the case of the interaction of a metallic ball with a dielectric plane but with no contact, and similar to the calculations of Durand, we have solved the problem of a metallic sphere in contact with a dielectric plane by using two infinite series of "image charges." 18,19 Details of the calculation are given in Appendixes A and B.

So, it has been possible to establish the relation between the total charge injected in the sphere, $Q_{\text {total }}$, and its surface potential $V_{l s}$ in the presence of the dielectric plane. Because of the experimental results (see Sec. IV), two cases have been considered: first, it was supposed that there was no charge implanted in the dielectric, all the injected charges $Q_{i}$ staying in the metallic sphere $\left(Q_{\text {total }}=Q_{i}\right)$, second, a part of the total charge was supposed to be reemitted ( $Q_{\text {total }}$ $-Q_{\text {reemitted }}=Q_{i}$ ) and a part of this reemitted charge was supposed to be introduced in the insulator. This implanted charge in the dielectric is assumed to be a single charge $Q_{t}$ (for trapped charge), placed just under the contact area.

In the first case, the relation between the injected charge $Q_{i}$ in the metallic sphere and its surface potential was

$$
V_{S}=-\frac{1}{4 \pi \epsilon_{0} R} \frac{A}{\ln (1-A)} Q_{i},
$$

with $A=\left(\epsilon_{r}-1\right) /\left(\epsilon_{r}+1\right), \epsilon_{r}$ is the dielectric constant of the insulator, and $R$ is the radius of the sphere. In this case, there is an attractive electrostatic force between the metallic sphere and the insulator plane, due to the attraction between "image" charges, it can be expressed by:

$$
F=\frac{1}{4 \pi \epsilon_{0} R^{2}}\left(\frac{A}{\ln (1-A)}\right)^{2} Q_{i}^{2} K_{\epsilon},
$$

where $K_{\epsilon}$ is a constant that depends only on the dielectric constant of the insulator (see Appendix A). For example, for a sapphire $\left(\epsilon_{r}=10\right)$, we have obtained $K_{\epsilon}=4.69$, for quartz $\left(\epsilon_{r}=4.3\right), K_{\epsilon}=0.91$.

In the second case, the charge $Q_{t}$ implanted in the insulator was supposed to be a single-point one and to be placed at a distance $d_{t}$ under the center of the contact area. In the

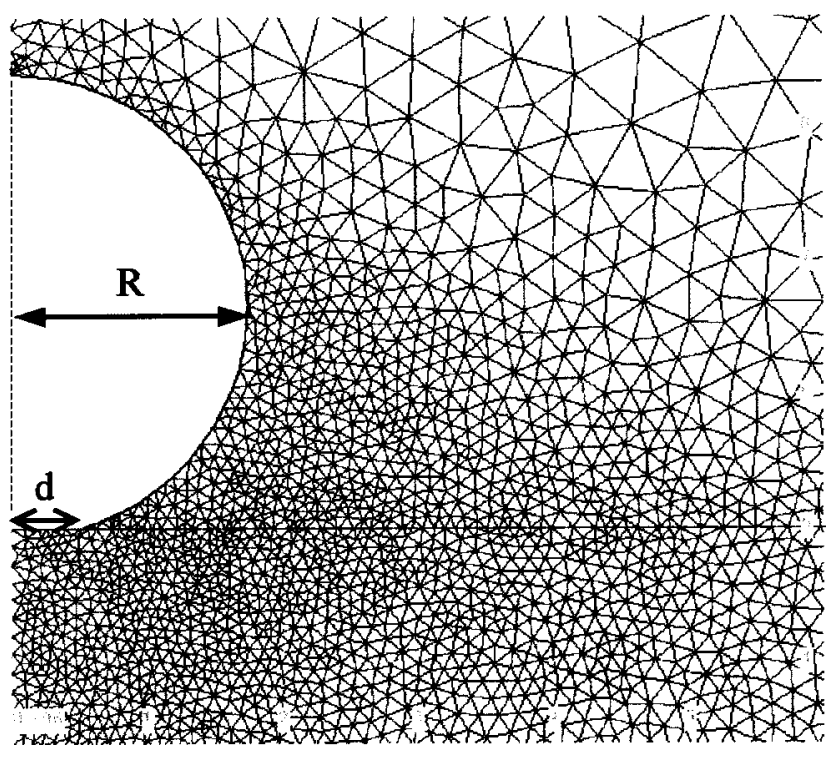

FIG. 2. Detail of the finite-element mesh (triangle of second order).

case of very high energetic electrons, the Monte Carlo simulation and the experimental results have shown that the penetration distance of electrons in the dielectrics was less than $1 \mu \mathrm{m} .{ }^{20}$ So, for the less energetic electrons of this experiment, it has been supposed that the implantation distance $d_{t}$ of the charges in the insulator was smaller than the radius of the metallic sphere $R, d_{t} \ll R$. Equation (3) gives the expression of the surface potential of the metallic sphere in the presence of the injected charge $Q_{i}$ staying in the sphere and the trapped charge $Q_{t}$ in the insulator:

$$
V_{s}=\frac{1}{4 \pi \epsilon_{0}}\left(\frac{-A}{\ln (1-A)} \frac{Q_{i}}{R}+\frac{2}{\epsilon_{r}+1} \frac{Q_{t}}{R}\right) .
$$

The contribution of the charge $Q_{t}$ to the surface potential of the sphere is the usual one established by Landau and Lifshitz. ${ }^{21}$ The electrostatic force follows

$$
F=\frac{1}{4 \pi \epsilon_{0} R^{2}}\left[\left(\frac{A}{\ln (1-A)}\right)^{2} Q_{i}^{2} K_{\epsilon}-\frac{2}{\epsilon_{r}+1} Q_{i} Q_{t}\right] .
$$

In the next section, the results of the analytical calculation will be compared with the potential values obtained by the finite-element method, for very thick insulating parallelepiped, and experimentally.

\section{B. Numerical model}

The effects of the presence of a metallic sphere in contact with an insulator sample lying on a grounded metallic plane were analyzed using a numerical model with axial symmetry presented in Fig. 2. For the numerical calculation, a sphere of radius $R=1.74 \mathrm{~mm}$ is put in contact thanks to a plane part (diameter of the plane part of the sphere: $d$ $=1.56 \mathrm{~mm}$ ) with an insulating disk of a diameter $D$ $=20 \mathrm{~mm}$ and of a thickness $h$. The dielectric constant of the disk is noted $\epsilon_{r}$, the insulator is placed on a grounded metallic plane, and the grounded walls of the SEM chamber are considered to stand at a distance of $100 \mathrm{~mm}$ (electrostatics 


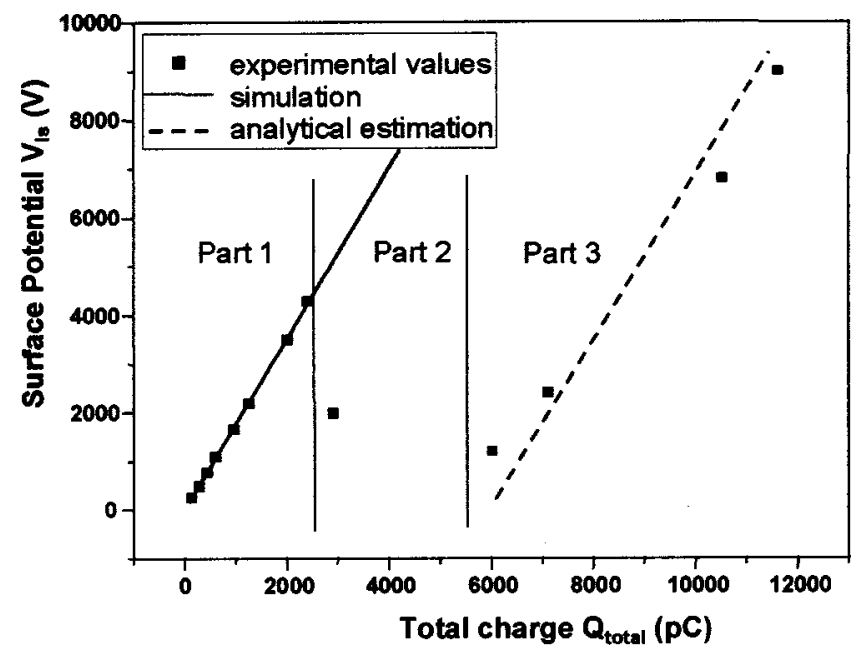

FIG. 3. Experimental curve of injection of electrons in a metallic sphere placed on an insulator sample.

results are minimally influenced by the distance of the SEM chamber as soon as the distance is high enough, this condition being verified in our SEM).

The numerical simulation was performed by solving electrostatic Laplace's equation with two-dimensional finiteelement package "Fissure" developed in CEGELY. ${ }^{10,22}$ The potential difference $V$ is applied between the sphere and ground. From the potential distribution, electric field $E$ is calculated along axis of sphere-disk-plane system. The capacitance between the metallic sphere and insulator disk is deduced from electrostatic energy calculated on the study domain.

\section{RESULTS AND COMPARISON WITH MODELING}

Figure 3 presents the variation of the surface potential $V_{l s}$ (measured experimentally as described in Sec. II C) versus the total charge $Q_{\text {total }}$ injected in the metallic sphere (precisely known because of the adaptation of the SEM to the SEMM method). As shown in Fig. 3, the experimental curve of the surface potential values versus the total injected charge in the sphere presents three parts: The first one is linear and has been attributed to an injection restricted to the sphere $\left(Q_{i}=Q_{\text {total }}\right)$, the second one is situated just after the slope break of the curve, and finally, the third part of the curve is approximately linear and parallel to the first one.

In Sec. IV A, the interpretation of the first part of the curve will be done with the help of the analytical and numerical results. A complementary measure of the injected charge in the sphere has also been done and will be developed in Sec. IV B.

The second and the third parts of the curve have been related to a charge transfer in the insulator or to a dielectric breakdown of the medium surrounding the sphere-plane system $\left(Q_{i}+Q_{t}=Q_{\text {total }}\right)$. This important point will be discussed in Sec. V.

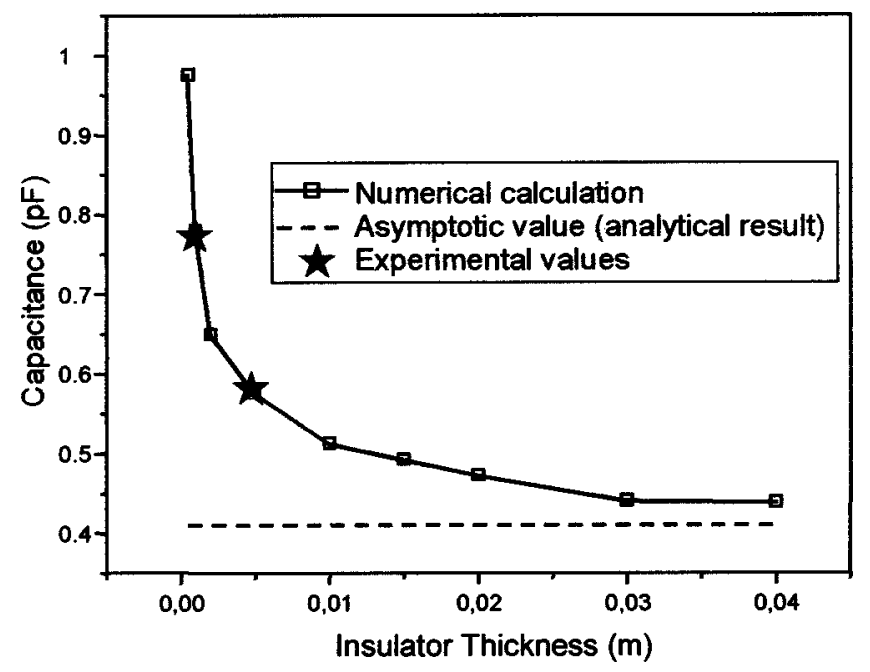

FIG. 4. Variation of the capacitance vs the sample thickness.

\section{A. Relation between the injected charges and the surface potential of the sphere}

The first part of the curve relating the surface potential $V_{l s}$ of the sphere and the total injected charge $Q_{\text {total }}$, has been precisely interpreted by considering that the total injected charge was staying in the metallic ball $\left(Q_{\text {total }}=Q_{i}\right)$. The linear variation of the potential versus the injected charge has been expressed in term of capacitance: $Q_{i}=C$ $V_{l s}$. In this case, the results of the numerical calculations of the capacitance are presented in Fig. 4 for its variation with the sample thickness, and in Fig. 5 for its variation with the dielectric constant.

The influence of the metallic holder just placed under the dielectric sample, is clearly evidenced in the Fig. 4. As expected, the simplest case of the semi-infinite dielectric plane obtained by analytical calculation appears to be the asymptotic case in the limit of very thick insulator. Note also the good agreement between experimental and calculation values. The experimental values were deduced from experi-

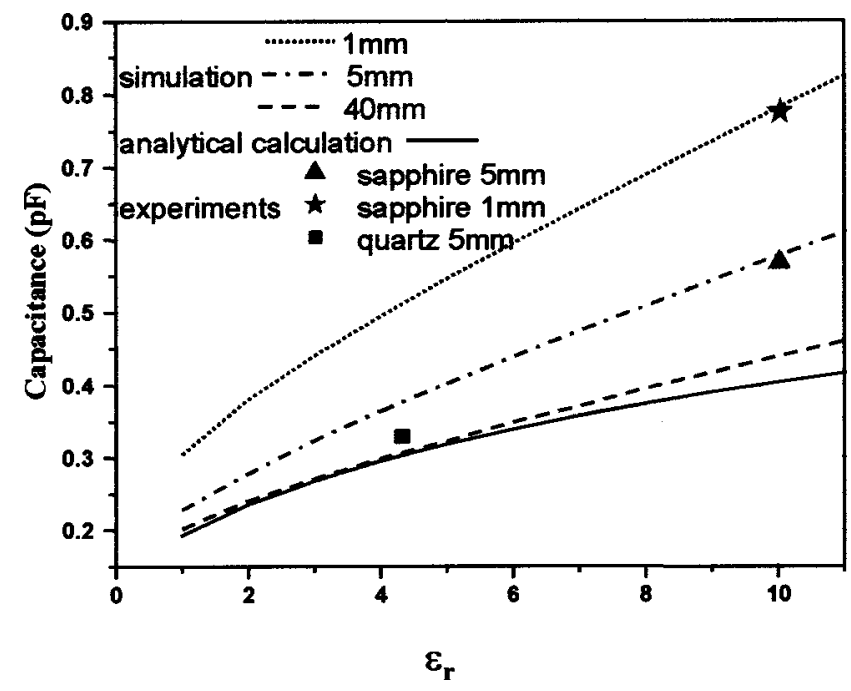

FIG. 5. Variation of the capacitance vs the dielectric constant and the sample thickness. 


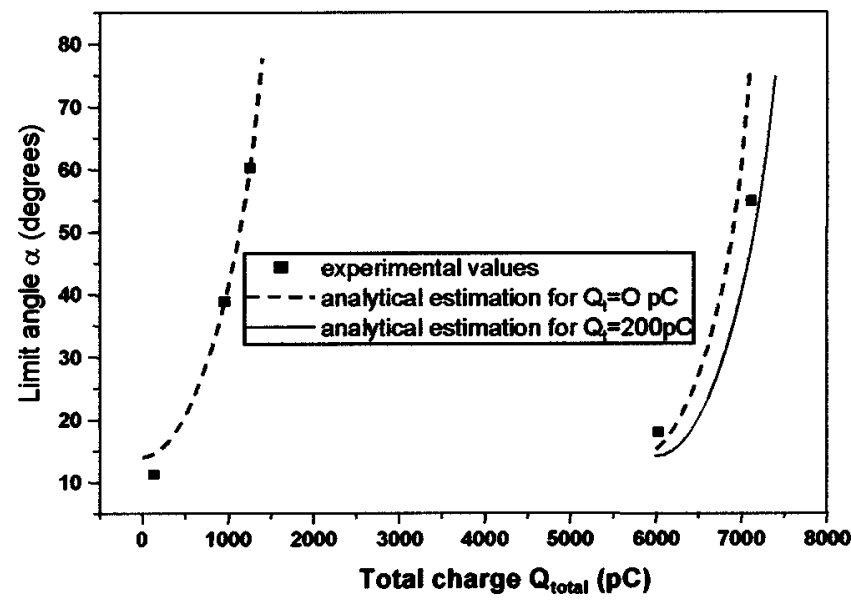

FIG. 6. Variation of the limit angle versus the total injected charge $Q_{\text {total }}$ and the quantity of charges implanted in the insulator $Q_{t}$.

ments on a sapphire sample with respective thicknesses of $4.94 \mathrm{~mm}$ and $1 \mathrm{~mm}$, and the calculations have been performed for a dielectric constant value, $\epsilon_{r}=10$, which is the commonly used average value for sapphire. Both the analytical and numerical calculations have revealed a dependence of the capacitance on the dielectric constant of the insulator. This fact has been experimentally confirmed as shown in Fig. 5. For both thicknesses of the sample, the agreement between experimental and simulation values is very good for sapphire $\left(\epsilon_{r}=10\right)$. In the case of quartz $\left(\epsilon_{r}=4.3\right)$, the agreement is not so good.

\section{B. Measure of the injected charge through the electrostatic force}

As shown in Fig. 1(b), the plane-sphere system can be tilted, forming an angle $\alpha$ with the horizontal line. To evidence the injected charge in the sphere (and in the dielectric for part 3 of the curve), the electrostatic force has been evaluated thanks to the measure of the limit angle $\alpha$ of sliding and ejecting the ball on the plane. For such an angle, the conditions of the equilibrium have been calculated as well as the sliding conditions with or without rolling. Experimentally, sliding has been observed first, before the ejection of the ball. The angle $\alpha$ of equilibrium break by sliding is obtained [cf. Eq. (5)] by writing the Newton's law and the relation between the normal and the tangential reaction thanks to the friction coefficient $k$,

$$
\sin \alpha-k \cos \alpha=\frac{k}{m g} F,
$$

with $m$ as the mass of the sphere, $g$ as the gravitational constant, and $F$ as the electrostatic force. Figure 6 illustrates the variation of the angle $\alpha$ with the increasing quantity of the total injected charge in the sphere. This result is probably the most striking one in the article because it underlines the importance of the electrostatic force compared to the weight of the sphere.

The calculated values of the angle $\alpha$ have been correctly fitted with the experimental values for small amounts of injected charges, i.e., when all the charges are supposed to stay

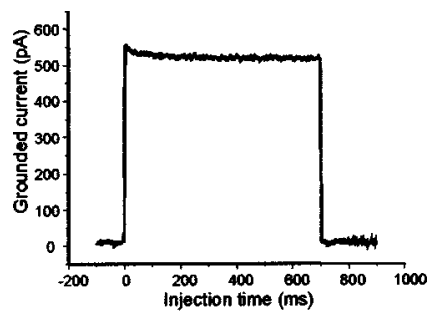

(a)

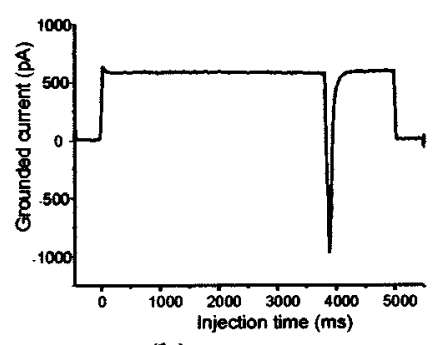

(b)

FIG. 7. Grounded current curve in the case of sapphire: (a) Total injection in the metallic ball and (b) Sharp decrease attributed to the release of electrons.

in the sphere (no breakdown or transfer). They have been obtained by using the electrostatic force given by Eq. (2) and the best fit has been reached for $k=0.25$. This result of the angle variation still confirms that all of the charges were staying in the sphere for relatively small amounts of injected charges. Similar to the curve relating the surface potential with the injected charges quantities (see Fig. 3), the limit angle also presents an abrupt change, after a first linear part, between 2000 and 6000 pC. Section V proposes explanations for these changes.

\section{DISCUSSION}

The break of the characteristic curves versus the total injected charges can be interpreted with two mechanisms confirmed by the experimental observations. In particular, the decrease of the electrostatic force, evidenced by the decrease of the limit angle (cf. Fig. 6) for the strong quantities of injected charges, can be interpreted either by a dielectric breakdown of the medium surrounding the sphere or (and) by injection of charges in the dielectric.

\section{A. Breakdown of the medium}

\section{Observation of electrons reemission}

The decreases of both electrostatic force and surface potential of the sphere are the first signs of a decrease in the quantity of charge in the sphere. Because of the high surface potential, it can be a release of electrons, for example by the breakdown of the medium. Another experimental observation, i.e., the form of the ground current curve, confirms this assumption. During the injection of electrons in the metallic sphere, it is possible to collect the ground current [current $I_{g}$ in Fig. 1(a)]. Considering that the leakage current is negligible, the ground current is principally an influence current created by the variation of the charge injected in the sphereplane system. For the injection in the metallic ball, a typical ground current curve is presented in Fig. 7(a). It has a form 
of step, corresponding to total injection (except secondary emission of the metal) of the charge in the metallic ball. Sometimes [cf. Fig. 7(b)], a sharp decrease of the ground current appears, it has soon been related to electron emission in the chamber of the SEM. ${ }^{12}$ The burst of the ground current appears randomly in part 2 of the curve shown in Fig. 3, but systematically in part 3 , it never appears in part 1 . In this particular arrangement, the burst of the ground current could be interpreted as a release of the electrons, for example, because of the breakdown of the medium.

In this case of strong trapping of charges in the sphere, the ground current curve can be used to evaluate the quantity of charges $Q_{\text {limit }}$ trapped in the sphere before the burst, thanks to the following relation: $Q_{\text {limit }}(t)=1 / \lambda \int_{0}^{t} I_{g} d t$, where $\lambda$ is an influence factor depending only on the parameters of the experiment (working distance, thickness, and dielectric constant) and $t$ is the burst time. So, it has been possible to determine an average limit value of injected charge in the sphere before the burst of the ground current. In the case of sapphire $(h=4.94 \mathrm{~mm})$, the average limit value is around $Q_{\text {limit }}=5890 \mathrm{pC}$.

\section{Breakdown initiation}

For high amounts of injected charges, the surface potential $V_{l s}$ of the sphere becomes higher. It can reach the value of the breakdown voltage of the medium surrounding the sphere. Several parameters of breakdown initiation are important, such as the shape of the sphere, the local roughness, the presence of metal-dielectric junction, and the surrounding medium. ${ }^{23}$ For example, Dascalescu et al. ${ }^{3}$ have shown that the radius of the sphere influences the limit of breakdown between a metallic sphere and a grounded plane in air. In our case, the medium is a high vacuum, with a pressure around $10^{-6}$ Torr during the experiment in the SEM. Because, in such an environment, the spark formation process is dominated by all the precedent parameters, it seems inadequate to define the breakdown strength of a high vacuum. However, in that pressure range, some researchers have reported breakdown fields on the order of 3-5 MV/m (comparable to air). The numerical calculation for a metallic sphere lying on a sapphire plane gives a maximum value of the electrical field at the contact between the dielectric plane and the metallic sphere $E_{\max }=987 \times V_{l S}$. With such a relation between the electric field and the surface potential, the breakdown of the medium, considering a dielectric strength of 5 $\mathrm{MV} / \mathrm{m}$ will be reached for $Q_{\text {total }}$ in the range of $3000 \mathrm{pC}$. It corresponds approximately to the limit of the linear part 1 of the Fig. 4.

However, it appears from a reading of the review of Farrall, that the dominant factor is the smoothness and the characteristics of the materials. ${ }^{23}$ Roughnesses of a small radius curvature or metal-dielectric junction cause field concentrations. If the radius is sufficiently small (for example, at the part plane side) the field can be high enough for field emission, which gets enough electrons out to provoke breakdown.

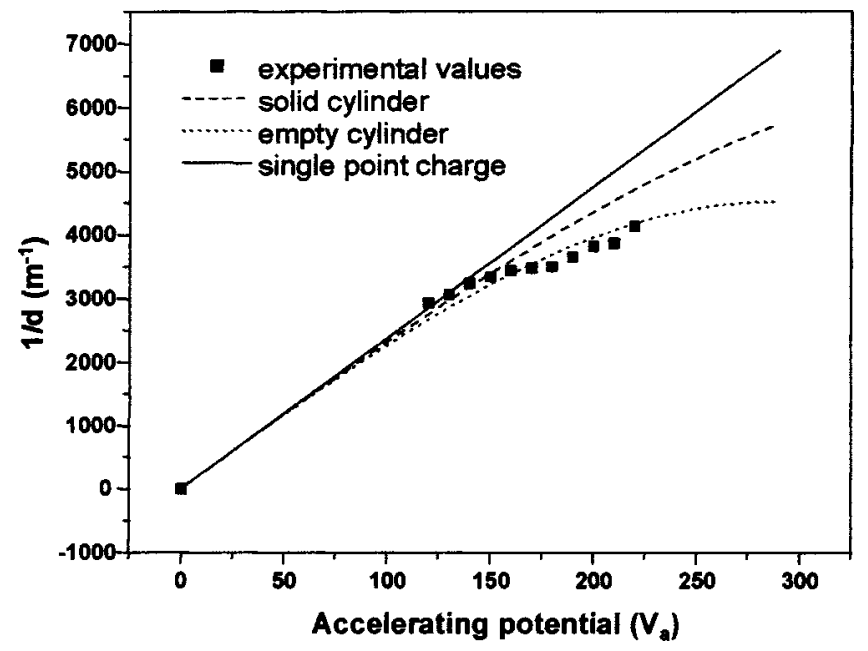

FIG. 8. Mirror $1 / d=f(V)$ plot of the charges injected in the dielectric plane. Comparison between experimental and calculated values, in a model of multipole approximation, for different geometries of the charge implantation.

\section{B. Injection of charges in the insulator}

Even if it seems very clear that breakdown occurs for high amounts of injected charges in the sphere, another experimental fact suggests a simultaneous injection of charges in the dielectric plane.

\section{Direct observation of electrical charges}

Indeed, after the injection of charges in the metallic ball, the system has been tilted, and the angle of sliding and ejecting the metallic sphere can be measured as shown previously. Immediately after ejecting the ball, the dielectric sample has been observed with the electron beam of the SEM and electrical charges have been imaged. At low magnification, their brightness has confirmed that they were electrons. These charges were located just in the previous position of the sphere. However, when reducing the observed area to those electrical charges, they have also led to the occurrence of a mirror image. The strong stability of the observed mirror with the increasing accelerating potential $V_{a}$ is a sign of an injection of the charges in the bulk, under the surface. For comparison, charges generated by triboelectrification, which can also be observed as shiny spots, are very unstable under the electron beam, and their corresponding mirror image cannot be focalized.

\section{Localization of the charges implanted in the dielectric}

Figure 8 presents the variation of the geometrical characteristic of the mirror versus the accelerating potential in the case of sapphire. The careful study of the mirror plot 1/d $=f(V)$ (obtained after the ejection of the metallic sphere) has led to an approximate quantity of trapped charges, in the insulator, in the range of $200 \mathrm{pC}$. As described in some previous studies, when the mirror is destabilized by a too high accelerating potential, some remaining charges have been observed, located on a circle whose diameter corresponds to the one of the plane part of the metallic sphere (noted $d$ in Fig. 2). This fact suggests that the injection has preferen- 
tially been effective at the metal-dielectric contact area, where the curvature radius of the junction was the smallest.

For verifying the preceding idea, the evolution of the curved part of the mirror plot has been interpreted in a model of multipole approximation. ${ }^{12}$ The curved part has been modeled with two different distribution geometries of the trapped charges. The first one is an empty cylinder of internal diameter $R$, height about $d_{t} \approx 1 \mu \mathrm{m}$ (cf. remarks on the $d_{t}$ value in Sec. III A) and thickness $e \approx d_{t} \approx 1 \mu \mathrm{m}$, too (external diameter $=R+e$ ). This geometry represents an injection only from the surrounding plane part of the sphere. The second geometry of the implanted charges in the insulator is a solid cylinder of diameter $R$ and height $d_{t}$, corresponding to the injection from the total junction area. Attard et al. ${ }^{24}$ have calculated the quadrupole contribution to the potential that can be written $V \propto\left[Q / r-\beta Q R_{0}^{2}\right]$ for a charge distribution with a cylindrical symmetry. $Q$ is the total charge, $R_{0}$ is the radius of the charge distribution, $r$ is relative to the center of charges: $r^{2}=x^{2}+z^{2}$ and $\beta$ is a parameter depending on the distribution shape. They have deduced an expression of the mirror plot: $1 / d \propto\left[V / A Q-2 \beta R_{0}^{2}\right]$, we have found $\beta$ $=1 / 12\left(6-d_{t}^{2} / R^{2}\right)$ for a solid cylinder of radius $R$ and height $d_{t}$. In the case of an empty cylinder, we have calculated $\beta=1+e / R-1 / 12 d_{t}^{2} / R^{2}+e^{2} / 2 R^{2}$. In our case, $R$ is in the range of millimeter and $e$ is in the order of micrometer, like $d_{t}$, so that $\beta \approx 1$. Figure 8 shows that the empty cylinder geometry fit better with the experimental curve, confirming that the injection in the insulator has been located in the circumference of the contact area.

\section{Proposed mechanism for high amount of injected charges in the metallic sphere}

Because of the previous experimental observations, a mechanism of charge transfer could be proposed in the case of a high amount of injected charges in the sphere. First, charges are injected in the metallic sphere only, the surface potential of the sphere increases following the linear law: $Q_{i}=C V_{l s}$. When the surface potential reaches a value sufficient to initiate the breakdown of the surrounding medium, the totality of the charges already present in the metallic sphere are ejected through an electron emission in the chamber and a small injection in the dielectric. This injection is favored by the point effect at the metal-dielectric junction. Clearly, the field emission takes a preferable place at the circumference of the part plane of the metallic sphere.

The situation is now the following: (i) the sphere has lost the majority of the previous injected charges noted $Q_{\text {reemitted }}$, the experimental value of $Q_{\text {reemitted }}$ corresponds to $Q_{\text {reemitted }}$ $=Q_{\text {limit }}=5890 \mathrm{pC}$ for sapphire, leading to a maximum electric field value in the range of $10 \mathrm{MV} / \mathrm{m}$ (cf. Sec. IV A 1). (ii) Some charges have been implanted in the dielectric, they will be noted $Q_{\text {trapped }}$ and their amount is around $Q_{\text {trapped }}$ $=200 \mathrm{pC}$. It is difficult to evaluate the number of electrons per unit area, because of the uncertainty about the location of their injection (cf. Sec. IV B 2). For instance, considering the empty cylinder hypothesis, that would represent roughly about $10^{17} \mathrm{e} / \mathrm{m}^{2}$. (iii) The charges issued from the continu- ation of the injection are injected once again in the metallic sphere only.

For part 3 of the curve linking the surface potential and the total injected charges, the surface potential follows the electrostatic Eqs. (3) and (4), with $Q_{i}=Q_{\text {total }}-Q_{\text {reemitted }}$. As shown in Fig. 3, the analytical calculation, issued from the precedent reasoning, correctly fits the experimental values. The differences between estimation and experiments could be related to the random part of the amount of reemitted charges.

In the same manner, for part 3 of Fig. 6, the limit angle $\alpha$ has been well fitted for an electrostatic force obtain by Eq. (2) with $Q_{i}=Q_{\text {total }}-Q_{\text {reemitted }}$, if the contribution of the implanted charges in the dielectric is neglected or by Eq. (4) with $Q_{i}=Q_{\text {total }}-Q_{\text {reemitted }}=Q_{\text {total }}-5890 \mathrm{pC}$ and $Q_{\text {trapped }}$ $=200 \mathrm{pC}$.

\section{CONCLUSION}

In the previous sections, qualitative and quantitative results have been given concerning the phenomenon occurring when a charged object is put in contact with an insulating material. From the fundamental point of view, the interaction mechanisms can be analyzed in three stages.

(1) For a low charged object, i.e., having a low surface potential, no charge transfer is observed between the two bodies: Electric charges remain in the charged object. For instance, in our steel-sapphire system, these limits are, respectively, a charge inferior to $2500 \mathrm{pC}$ and a surface potential inferior to $4.5 \mathrm{keV}$.

(2) Beyond these limit conditions, breakdown might appear but presents an uncertain feature. It may occur when the potential and the electric field are sufficient to induce a breakdown, starting from favorable sites (for example, microasperities) or induced by circumstances (inadequate surface decontamination, etc.). ${ }^{25}$ Then, the charges present in the object suddenly spread out in the surrounding space.

(3) Beyond a critical quantity of charges $\left(Q_{i} \geqslant 6000 \mathrm{pC}\right)$ in our experiments), the breakdown appears systematically. This breakdown is not only characterized, as previously described, by a release of charges, but also by a transfer and trapping of charges into the insulating material. This trapping, as proven by the mirror effect, corresponds to a stable and deep space charge (about $200 \mathrm{pC}$ in our case) located in the bulk, under the object/material interface. The effect of the injection mode of charges (speed, step by step, etc.) on the breakdown initiation is in progress because it seems to be a determinant parameter.

From the technological point of view:

(1) An electrostatic adhesion between the two bodies in contact is obvious and can induce triboelectrification effects. ${ }^{6}$

(2) Uncontrolled breakdown causes a high electric field which favors damaging of systems, for example, electronic systems, present in the surrounding impact zone.

(3) The transfer of electric charges into the insulating material creates a space charge which can change the surface 
properties (surface energy, adhesion, friction coefficient, wetability, etc.), ${ }^{6,26}$ the bulk properties (toughness, breakdown resistance, etc.), ${ }^{26-28}$ and their release is a determining factor for the aging of insulating materials.

\section{ACKOWLEDGMENTS}

The authors wish to acknowledge O. Pauhle and J. Vallayer for preliminary work, J. Peyrelasse for mechanical calculations, and C. Le Gressus and J. Cazaux for fruitful discussions. This work was partially supported by CEA Le Ripault (Monts, France).

\section{APPENDIX A}

The analytical calculation of the surface potential of the metallic sphere can be found in Durand's book in the case of a metallic plane. ${ }^{17}$ In our case of an insulator plane and of contact between the sphere and the plane, the positions of the image charges remain the same, however, a coefficient $A$ $=\left(\epsilon_{r}-1\right) /\left(\epsilon_{r}+1\right)$ has to be introduced because of the dielectric-vacuum interface.

In this first appendix, the following system is considered: A metallic sphere, containing an electrical charge $Q_{i}$ and lying on a dielectric plane (dielectric constant $\left.\epsilon_{r}\right)\left(Q_{i}\right.$ $\left.=Q_{\text {total }}\right)$, but containing no charge. We have adapted Bacchetta's formalism to our problem. ${ }^{19}$ The field in a vacuum and so the value of the potential of the metallic sphere, can be seen as created by a double series of charges $q_{i}$ in the sphere and $q_{i}^{\prime}$ in the dielectric. The values and positions of the charges are defined by the recurrence relations:

$$
\begin{cases}q_{i+1}=A^{i} q_{1} \frac{1}{i+1}, & i>0 \\ q_{i}^{\prime}=-A^{i} q_{1} \frac{1}{i}, & i>0 \\ \Delta_{i+1}=\frac{i}{i+1} R, & i>0\end{cases}
$$

where $R$ is the radius of the sphere, $\Delta_{i}$ is the distance of the charge $q_{i}$ to the center of the sphere. The charge $q_{i}^{\prime}$ is symmetrical to $q_{i}$ in relation with the interfacial plane.

Because of the experimental conditions, it has been supposed that the injected charge $Q_{i}$ in the sphere was known, and the potential was unknown. So, by writing $Q_{i}$ $=\sum_{i \geqslant 1} q_{i}$, it comes: $q_{1}=-A / \ln (1-A) Q_{i}$.

The potential of the sphere has been evaluated in its center:

$$
V_{s}=\sum_{i=1}^{\infty} \frac{q_{i}}{4 \pi \epsilon_{0}\left(R-\Delta_{i}\right)}=-\frac{1}{4 \pi \epsilon_{0} R} \frac{A}{\ln (1-A)} Q_{i} .
$$

The electrostatic force undergone by the sphere is assumed to be the sum of the elementary Coulombian forces between two single-point charges, one situated in the sphere, the second in the dielectric.

$$
\begin{aligned}
F & =\frac{1}{4 \pi \epsilon_{0}} \sum_{i} \sum_{j} \frac{q_{i} q_{j}^{\prime}}{\left(2 R-\Delta_{i}-\Delta_{j}\right)^{2}} \\
& =\frac{1}{4 \pi \epsilon_{0} R^{2}}\left(\frac{A}{\ln (1-A)}\right)^{2} Q_{i}^{2} K_{\epsilon},
\end{aligned}
$$

where $K_{\epsilon}=\Sigma_{i} \Sigma_{j} A^{(i+j)-1} i j /(i+j)^{2}$. The constant $K_{\epsilon}$ can be evaluated numerically because of the rapid convergence of the double infinite sums.

\section{APPENDIX B}

In this second appendix, the following system is considered: a metallic sphere (radius $R$ ), containing an electrical charge $Q_{i}=Q_{\text {total }}-Q_{\text {reemitted }}$ and lying on a dielectric plane (dielectric constant $\epsilon_{r}$ ) but containing a trapped charge $Q_{t}$. As specified in Sec. III A, the charge $Q_{t}$ implanted in the insulator was supposed to be a single-point one and to be placed at a distance $d_{t}$ under the center of the contact area.

The principle of the calculation is the same as in the previous appendix. A first double series of charges $q_{i}$ in the sphere and $q_{i}^{\prime}$ in the dielectric is considered to evaluate the effect of the charge $Q_{i}$ located in the sphere. The charges $q_{i}$ and $q_{i}^{\prime}$ and their respective positions $\Delta_{i}$ are defined by the previous recurrence relation (6) except for the value of $q_{1}$.

A second double series of charge $q_{i}^{\prime d}$ in the dielectric and $q_{i}^{d}$ in the sphere is used to calculate the field and potential due to the implanted charge in the dielectric $Q_{t}$. If the point charge $Q_{t}$ is situated at a distance $R+d_{t}$ vertically to the center of the sphere, the values and positions $\Delta_{i}^{d}$ of the charges $q_{i}^{\prime d}$ and $q_{i}^{d}$ are defined by the following recurrence relations:

$$
\left\{\begin{array}{l}
q_{i+1}^{d}=A^{i} q_{1}^{d} \frac{\left(R+d_{t}\right)}{\left[(i+1) R+d_{t}\right]}, \quad i>0, \\
q_{i}^{\prime d}=-A^{i} q_{1}^{d} \frac{\left(R+d_{t}\right)}{\left[i R+d_{t}\right]}, \quad i>0 \\
\Delta_{i+1}^{d}=\frac{R\left(i R+d_{t}\right)}{\left[(i+1) R+d_{t}\right]}, \quad i>0
\end{array}\right.
$$

with $q_{1}^{d}=2 / \epsilon_{r}+1 Q_{t}$. In this case, the value of $q_{1}$ is defined by the relation: $Q_{i}=\Sigma_{i \geqslant 1} q_{i}+\sum_{i \geqslant 1} q_{i}^{d}$. In the approximation $d_{t} \ll R$, the potential value of the sphere (evaluated in its center):

$$
\begin{aligned}
V_{s} & =\sum_{i=1}^{\infty} \frac{q_{i}}{4 \pi \epsilon_{0}\left(R-\Delta_{i}\right)}+\sum_{i=1}^{\infty} \frac{q_{i}^{d}}{4 \pi \epsilon_{0}\left(R-\Delta_{i}^{d}\right)} \\
& =-\frac{1}{4 \pi \epsilon_{0} R}\left[\frac{-A}{\ln (1-A)} Q_{i}+\frac{2}{\epsilon_{r}+1} Q_{t}\right] .
\end{aligned}
$$

The electrostatic force undergone by the sphere is assumed to be the sum of the elementary Coulombian forces between two single-point charges, one situated in the sphere, the second in the dielectric. 


$$
\begin{aligned}
F= & \frac{1}{4 \pi \epsilon_{0}} \sum_{i} \sum_{j} \frac{q_{i} q_{j}^{\prime}}{\left(2 R-\Delta_{i}-\Delta_{j}\right)^{2}} \\
& +\frac{1}{4 \pi \epsilon_{0}} \sum_{i} \sum_{j} \frac{q_{i}^{d} q_{j}^{\prime}}{\left(2 R-\Delta_{i}-\Delta_{j}\right)^{2}} \\
& +\frac{1}{4 \pi \epsilon_{0}} \sum_{i} \sum_{j} \frac{q_{i} q_{j}^{\prime d}}{\left(2 R-\Delta_{i}-\Delta_{j}\right)^{2}} \\
& +\frac{1}{4 \pi \epsilon_{0}} \sum_{i} \sum_{j} \frac{q_{i}^{d} q_{j}^{\prime}{ }^{d}}{\left(2 R-\Delta_{i}-\Delta_{j}\right)^{2}} .
\end{aligned}
$$

For $d_{t} \ll R$, it comes:

$$
F=\frac{1}{4 \pi \epsilon_{0} R^{2}}\left[\left(\frac{A}{\ln (1-A)}\right)^{2} Q_{i}^{2} K_{\epsilon}-\frac{2}{\epsilon_{r}+1} Q_{i} Q_{t}\right],
$$

where $K_{\epsilon}=\Sigma_{i} \Sigma_{j} A^{(i+j)-1} i j /(i+j)^{2}$, as in Appendix A.

${ }^{1}$ B. Vayner, J. Galofaro, and D. Ferguson, Proceedings of the Seventh Spacecraft Charging Technology Conference, 23-27 April, 2001, ESAESTEC Noordwijk, The Netherlands, edited by R. A. Harris (ESA Publication Division, Noordwijk, 2002).

${ }^{2}$ S. Banerjee and M. K. Mazumder, IEEE Trans. Ind. Gen. Appl. 36, 46 (2000).

${ }^{3}$ L. Dascalescu, P. Ribardière, C. Duvanaud, and J.-M Paillot, J. Electron. 47, 249 (1999).

${ }^{4}$ W. D. Greason, Electrostatic Discharge in Electronics (Research Studies Press, London, 1987).

${ }^{5}$ J. Lowell and A. C. Rose-Innes, Adv. Phys. 29, 947 (1980).

${ }^{6}$ C. Guerret-Piécourt, S. Bec, and D. Tréheux, C. R. Acad. Sci., Ser. IV 2, 761 (2001).

${ }^{7}$ X. F. Zong, C. P. Shen, S. Liu, Y. Chen, and R. Zhang, Phys. Rev. B 54, 139 (1996).
${ }^{8}$ A. M. Stoneham, IEEE Trans. Dielectr. Electr. Insul. 4, 604 (1997).

${ }^{9}$ G. Damamme, C. Le Gressus, and A. De Reggi, IEEE Trans. Electr. Insul. 4, 558 (1997).

${ }^{10}$ B. Vallayer, G. Blaise, and D. Treheux, Rev. Sci. Instrum. 70, 3102 (1999).

${ }^{11}$ H. J. Wintle, J. Appl. Phys. 86, 5961 (1999).

${ }^{12}$ J. Bigarré, C. Attard, P. Hourquebie, and J. Matallana, IEEE Trans. Dielectr. Electr. Insul. 8, 942 (2001).

${ }^{13}$ F. Vila and G. Touchard, J. Electron. 40, 121 (1997).

${ }^{14}$ J. Vallayer, C. Jardin, and D. Tréheux, Opt. Mater. 16, 329 (2001).

${ }^{15}$ O. Hachenberg and W. Brauer, Adv. Electron. 11, 413 (1959).

${ }^{16}$ A. Berroug, Proceedings of the IEEE Annual Report Conference on Electrical Insulation and Dielectric Phenomena, Minneapolis, MN, October 19-22 (IEEE, New York, 1984), pp. 97-100.

${ }^{17}$ W. R. Smythe, Solutions to Problems in Static and Dynamic Electricity (McGraw-Hill, New York, 1968).

${ }^{18}$ H. Bachetta, Ph.D. thesis, Université d'Orsay, 1999.

${ }^{19}$ H. Bachetta, J.-P. Beghe, A. Berthault, P. Carles, and J.-C. Niepce, Rev. Sci. Instrum. 70, 2105 (1999).

${ }^{20}$ C. Attard and J. P. Ganachaud, Phys. Status Solidi A 199, 455 (1997).

${ }^{21}$ L. Landau and E. M. Lifshitz, Electrodynamics of Continuous Media (Pergamon, Oxford, 1960), Vol. 8, pp. 55-65.

${ }^{22}$ N. Burais, L. Krahenbuhl, and A. Nicolas, Proceeding of the International Conference on Electrical Machines, Lausanne, Switzerland, September 18-21, 1984.

${ }^{23}$ G. A. Farral, Proc. IEEE 61, 1116 (1973).

${ }^{24}$ C. Attard, J. Bigarré, and P. Hourquebie, Proceeding of the Second Conference on Electrostatics, Montpellier, France, July 10-11, 2000, p. 77.

${ }^{25}$ G. A. Farral, F. G. Hudda, and R. H. Johnston, J. Appl. Phys. 50, 3608 (1979).

${ }^{26}$ A. M. Stoneham and P. W. Tasker, J. Phys. C 18, 543 (1985).

${ }^{27}$ A. M. Stoneham, Supplément de la revue "Le Vide: science, technique et applications," (Societé Française du Vide, Paris, 1998), pp. 1-8.

${ }^{28}$ J. Vallayer et al., Matériaux et Techniques 7, 15 (2000). 